\title{
Fabrication of Integrated Optical Mode Field Adapters at the End of Single/Multimode Fibers Using Fiber Splicing Machine
}

\author{
U.H.P. Fischer ${ }^{*}$, T. Windel, S. Hemrungrote and V. Giannoglou \\ Harz University of Applied Sciences, Friedrichstr. 57, 38855 Wernigerode, Germany
}

\begin{abstract}
In this paper we present the fabrication of optical mode field adaptors for fiber optical communications using fiber splicing machine in special newly developed modus. We developed mode field transformers with radii from $5 \mu \mathrm{m}$ to $80 \mu \mathrm{m}$ the end of standard singlemode and multimode glass fibers, characterized by far field and near field methods. Easy and reliable fabrication of the radii with low standard deviation $\sigma=0.16-0.97 \mu \mathrm{m}$ or better than $3,2 \%$ relative to the mean radius has been achieved.
\end{abstract}

Keywords: Fiber-chip coupling, fiber tapers, lensed fibers, optical mode field adaptation, integrated optical mode field transformers.

\section{INTRODUCTION}

The demand for high-speed digital communication such as data, video, and the broadband internet increases, the required throughput of the modules in communication systems will also increase [1]. Fast transmitter and receiver modules are basic elements of these systems, which should be able to transmit terabits/s of information via the fiber. Such technologies in turn rely strongly on advanced optoelectronically technologies, and the progress made in integrated optics. With rapidly increasing market demand for the use of these technologies and access to the information highway, the next challenge in optical communications is to provide high coupling efficiency in stable modules at affordable prices [2].
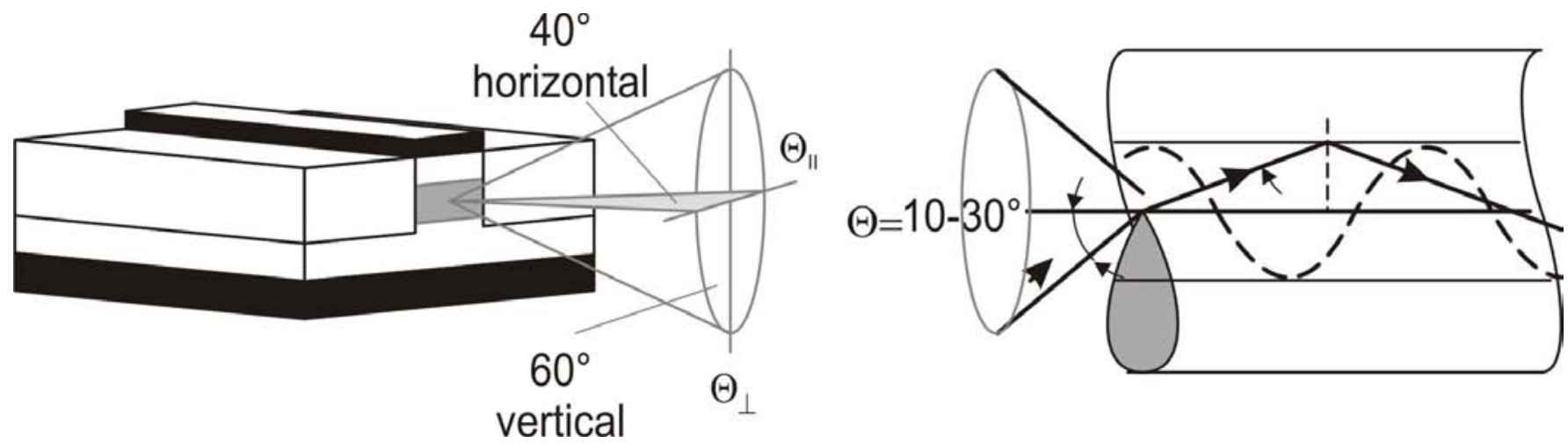

Fig. (1). Optical far field of SMF and laser diodes.

\section{OEIC-FIBER COUPLING}

One important item in all optical transmission systems is the necessity to couple a maximum amount of light from a

*Address correspondence to this author at the Harz University of Applied Sciences, Friedrichstr. 57, D-38855 Wernigerode, Germany; Tel: +49 3943 659351; Fax: +49 3943 659399; E-mail: ufischerhirchert@hs-harz.de single mode laser diode into a standard monomode fiber (SMF). Additionally, the coupling from SMF into photo diodes and the coupling of light from/into SMF to passive waveguides in InP or GaAs devices must be performed with high efficiency.

For low cost coupling a butt fiber is normally used, which is fixed with glue in front of the opto-electronic integrated circuit (OEIC) facet. For lasers and passive waveguides this technique allows only $10-15 \%$ coupling efficiency because of the great mode field mismatch between butt ended cleaved standard SMF and integrated waveguides (Fig. 1).

Saruwatari has shown the analytical calculation of the SMF to OEIC coupling problem in detail $[3,4]$. The optical 


$$
\mathrm{w}_{0}=a\left[0.65+\frac{1.619}{V^{3 / 2}}+\frac{2.879}{V^{6}}\right]
$$

with the fiber core radius a. The mode field radius for SMF at the wavelength of $1310 \mathrm{~nm}$ is $4.65 \mu \mathrm{m}$. The coupling efficiency $h$ between the two Gaussian beams of the two adjacent waveguides is disturbed by phase matching problems and misalignments in the lateral axis $\left(\mathrm{x}_{0}\right)$, longitudinal axis $(\mathrm{z})$ and in the angular offset $(\Theta)$. With this the efficiency can be calculated to be:

$$
\eta=\kappa \exp \left(-\kappa\left\{\frac{x_{0}}{2}\left(\frac{1}{w_{1}^{2}}+\frac{1}{w_{0}^{2}}\right)+\pi \Theta^{2}\left(\frac{w_{1}^{2}(z)+w_{0}^{2}}{2 \lambda^{2}}\right)-\frac{x_{0} \Theta}{w_{1}}\right\}\right)
$$

using

$\kappa=\frac{4 w_{1}^{2} w_{0}^{2}}{\left[w_{1}^{2}+w_{0}^{2}\right]^{2}+\lambda^{2} z^{2} / n \pi^{2}}$

and

$$
w_{1}^{2}(z)=w_{1}^{2}\left\{1+\left[\frac{\lambda z}{n \pi^{2} w_{1}^{2}}\right]^{2}\right\}
$$

and $\lambda$ as the wavelength. For no longitudinal, angular and lateral offset the efficiency is only a function of the difference of the two mode fields, $w_{0}$ and $w_{1}$ respectively. The calculation of typical mode field diameter of a laser diode at $1300 \mathrm{~nm}\left(w_{0}=1.5 \mu \mathrm{m}\right)$ and a SMF of $w_{1}=4.65 \mu \mathrm{m}$ results in a coupling loss of $4.557 \mathrm{~dB}$ without any misalignment can be easily performed by Eq. (5):

$\eta_{d B}=10 \log _{10}(\eta)=10 \log _{10}(\kappa)$

using $\mathrm{z}, \mathrm{x} 0, \Theta=0$, which means no additional dislocations between the two waveguides. This is a theoretical value; in practice a coupling efficiency of only $5-15 \%$ can be realized.

For higher coupling efficiencies one has to adapt the mode fields of the OEIC and the SMF for good overlap. The numerical aperture (NA) of the SMF of 0.15 or GI-MMF of 0.21 is low in comparison to an InP DFB-Laser of 0.3-0.6. A lens or a system of lenses can perform this adaptation of the mode fields. A commonly used system consists of two lenses with a parallel beam part in between where an optical isolator can be inserted to prevent back reflections into the emitting device. Commercial solutions for high-speed networks for more than $10 \mathrm{Gbit} / \mathrm{s}$ transmission rates typically use these two lens systems with an optical isolator with $60 \mathrm{~dB}$ isolation and are available from different suppliers. The relatively high cost of the components, in combination with a high amount of work time handling many discrete parts, presents a great disadvantage and increases packaging cost. Taper lenses (also called fiber tapers) offer very low reflections of less than $50 \mathrm{~dB}$ because of their end radii. Polarization independent all fiber inline isolators can be placed directly behind coupling region to fulfill special specs of high speed systems.

The advantages of these fiber tapers are the small dimensions and the integration into the end of the SMF which results in a very small opto-electronically package. The most disadvantages are the high sensitivity to small mechanical dislocations. $0.3 \mu \mathrm{m}$ lateral dislocation results in ca. $2-3 \mathrm{~dB}$ optical loss.

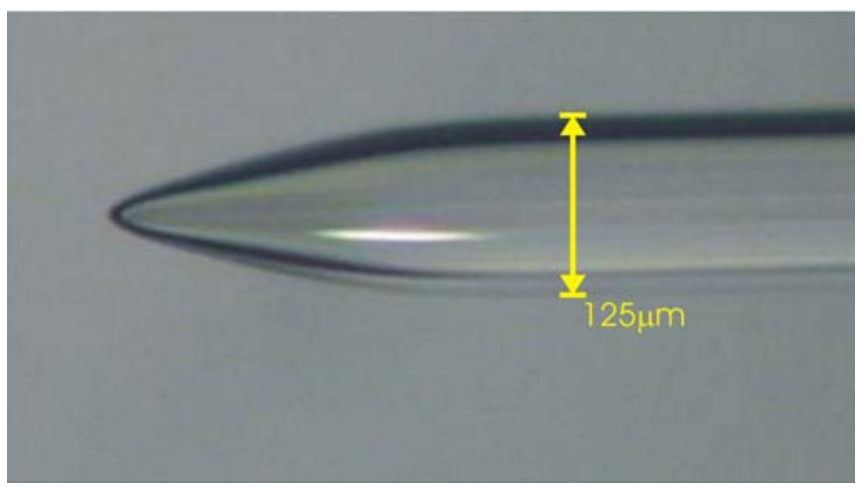

Fig. (2). Fiber taper lens.

The low efficiency can also be overcome by an adaptation of the optical mode fields with our developed mode field transformers, so called fiber taper lenses (Fig. 2). Parameters are developed to shape the radius of the fiber end for all glass fiber types of SMF, step index (SI) MMF or graded index (GI) MMF from $5 \mu \mathrm{m}$ up to $80 \mu \mathrm{m}$.

\section{INTEGRATED OPTICAL MODE FIELD TRANS- FORMER}

Reducing the cost of the packaging could be established by using a micro lens at the end of the SMF, which is called mode field transformer or fiber taper, respectively [6]. We are fabricating these fiber tapers by melting the fiber in a RF-plasma and drawing it in longitudinal direction (Fig. 3). Other approaches to form such lenses are by photolithography, etching, micromachining or dipping into molten glass and fusing [7-9].

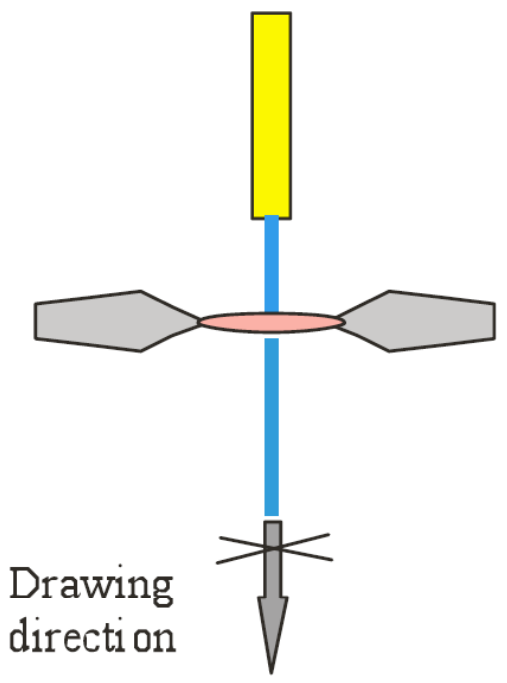

SMF cable

Electrodes for fiber heating Drawing fiber

\section{$\mathrm{Y}, \mathrm{X}$ adjusting of drawing fiber}

Fig. (3). Schematic view of the drawing process.

For the realization of the fiber tapers a specially adapted fiber splicing machine of Fujikura was used. The splicing machine was modified mechanically and in software to get a long drawing length of the z-axis. The ends of two prefabricated butt-ended glass fibers are fusion spliced in a first step. In the second step an additional heat pulse is added by current flow between two electrodes activating a RF-plasma and pulls one of the fiber ends with a distinct velocity to the 
outside direction. Here, the splice is melting and drawn apart. As a function of several parameters, a lens like fiber end can be realized (Fig. 4).

Several parameters must be controlled very precise to form the desired shape and radius of curvature of the fiber lens:

- Heat injection power

- Injection time

- $\quad$ Pulling speed

- $\quad$ Pulling length

The full list of parameters and there timetable for one tapering process is depicted in Fig. (5).

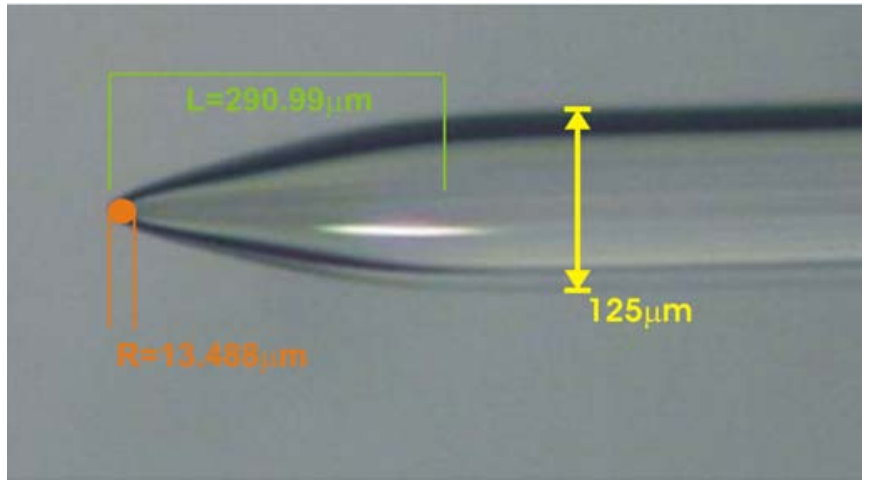

Fig. (4). Parameters for taper lens analysis.

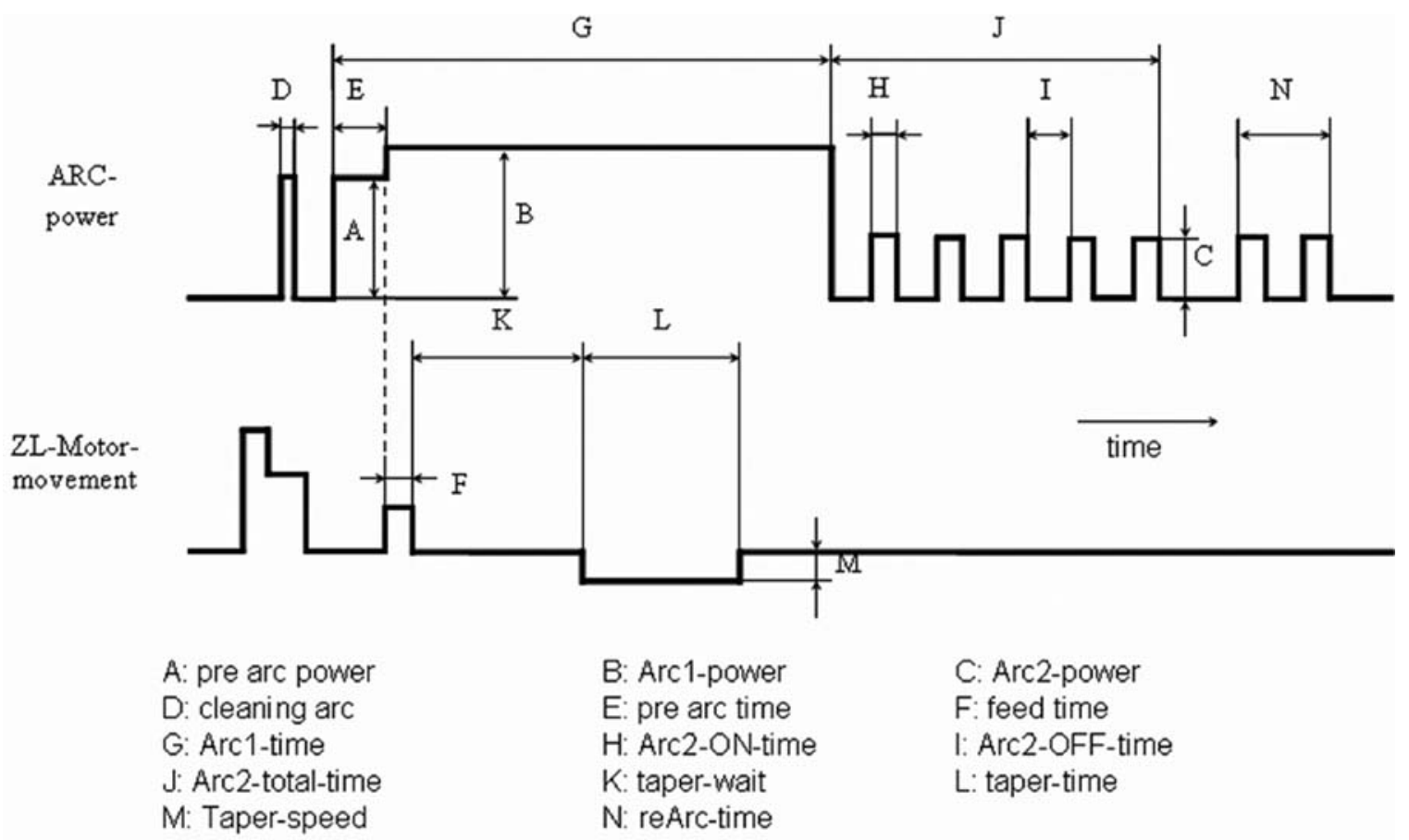

Fig. (5). Parameters to control the tapering process.

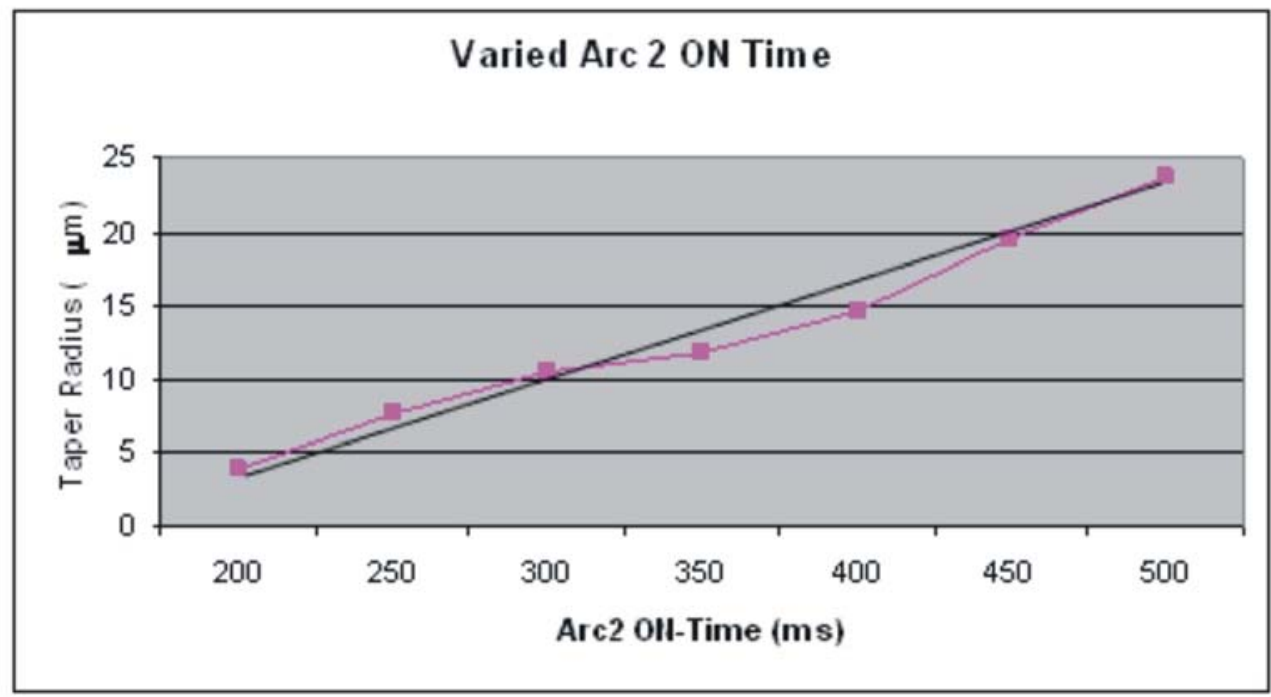

Fig. (6). Variation of arc time in correlation to lens radii. 
By changing one or more of these parameters you obtain different radii of the fiber taper. For example: by extending the Arc2-ON-time there is more introduced power to the glass, which impacts a higher temperature into the device. This results in a larger taper radius (Fig. 6).

All kinds of glass fibers can be processed $(9 \mu \mathrm{m} / 50 \mu \mathrm{m} / 62.5 \mu \mathrm{m})$, as well step index (SI) profiles, as graded index multimode fibers (GI-MMF). For MMF fibers, the lens function will also be effective, but with larger spotsize in comparison to the lens radius.

The end of the glass fiber is formed conical and works like a mode field transformer. Using a special lens radius, an optimum adaptation to the mode field of the coupling OEIC to the fiber can be realized.

After the drawing process the following mechanical and optical parameters of the lens are quantified:

- $\quad$ Length of pulled region

- Radius of fiber tip end

- Symmetry

- Optical mode field/spot size

As it can be noticed in Fig. (7), fabrication with low standard deviation $\sigma=0.16 \mu \mathrm{m}$ is achieved for 50 samples with $\mathrm{r}=10.1 \mu \mathrm{m}$ mean radius, which corresponds to less than $2 \%$ relative to the mean. This good result is because all samples were manufactured of one type of fiber.

To adapt the outline of the fiber taper, the optical mode field of the laser diode must be characterized very exactly. In the following different methods for mode field acquisition are described and a new technique will be proposed.

\section{MODE-FIELD MEASUREMENTS}

There are several methods to characterize the optical mode field of photonic devices [10-12]. The most used method is the so-called far field method [13, 14]. Here the optical far field is measured by a scanning photodiode, which is rotated around the output side of optical waveguide.
The second often used method is using a microscope objective to enlarge the surface spot of the waveguide front to a camera system. This method is called in literature the near field technique $[5,15]$. To overcome the disadvantages of the conventional methods, we developed a new set-up for scanning the optical field linearly without any additional optics or $\mu \mathrm{m}$-precise adjustment of the DUT. The new method working in the far field is called Medianfieldmethod. A closer description of this conversion and the measurement set-up can be found in a previously published paper [16].

\section{FIELD MEASUREMENT RESULTS}

For calibrating the Medianfield-method, we used a reference-SMF of NIST (National Institute of Standardization, Boulder, USA), where the optical field width $w_{0}$ was wellknown $\left(2 w_{0}=9.312 \mu \mathrm{m} @ 1310 \mathrm{~nm}\right.$ and $10.489 \mu \mathrm{m} @$ $1550 \mathrm{~nm}$ at $\left.1 / \mathrm{e}^{2}\right)$. As it can be seen in Fig. (8), the 2dimensional far field distribution is shown, while the field distribution can be well approximated by Gaussian function. Our results of the mode field diameter of $10.34 \mu \mathrm{m} \pm 3 \%$ @ $1550 \mathrm{~nm}$ is in good agreement (error of 1.45\%) to the given NIST-value [17]. As an example of a symmetrical field distribution of the fabricated fiber tapers, Fig. (9) indicates the far field of the SMF with lensed end.

For several lens radii from $8 \mu \mathrm{m}$ up to $50 \mu \mathrm{m}$ at the end of SMF-fibers the optical mode fields by the far field and Medianfield-technique were measured. In Table 1 the realized fiber lenses with standard deviation and medium lens radius are shown. For smaller radii the mean value of the measured lens shape becomes larger than expected. A possible reason for this behaviour can be the caused by the strong surface forces of the fused glass, which stretches short radii more than larger ones.

In Fig. (10) the measured and the calculated relation - by a thick lens correction by Ladany - of fiber end radius and acceptance angle are depicted [18]. For each radius five individual devices are drawn and characterized.

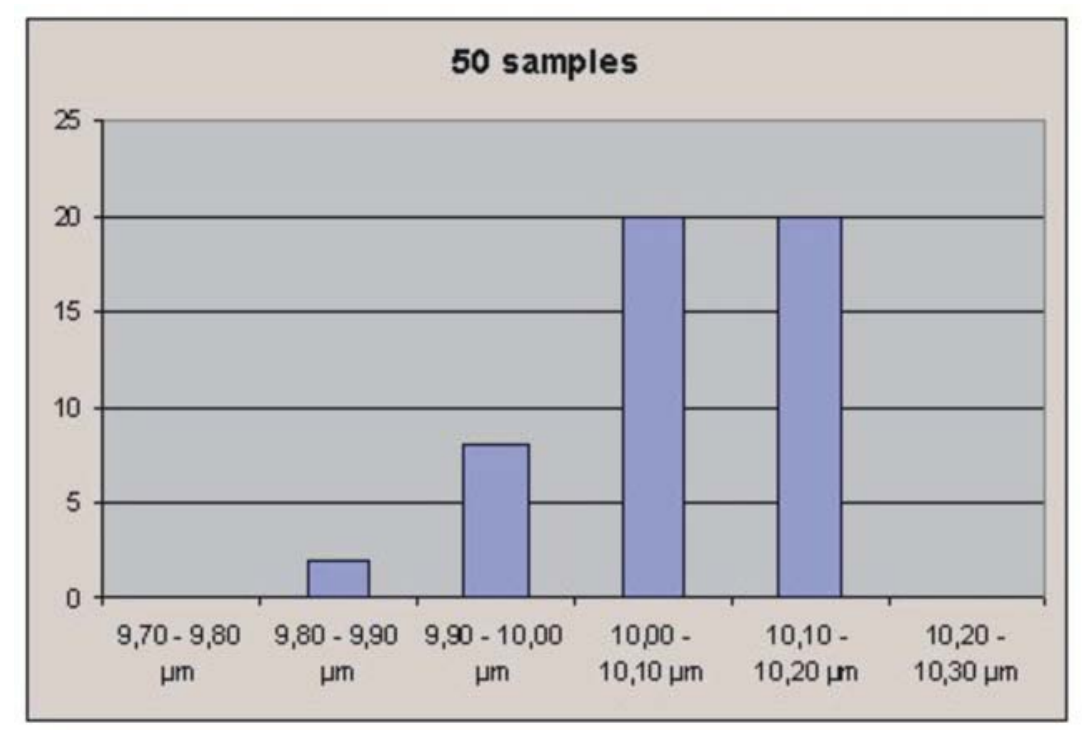

Fig. (7). Histogram of the radius distribution of a sample of 50 tapers drawn with identical fibers and machine parameters (mean radius $\mathrm{r}=10.1 \mu \mathrm{m}$, standard deviation $\sigma=0.16 \mu \mathrm{m})$. 
It can be noticed from Fig. (10) that the relation between acceptance angle and lens radius shows a good agreement with the calculated data from Ladany including thick lens correction factors. The good agreement with the calculation is effected by that the fiber core is only slightly degenerated from its original diameter of $8.5 \mu \mathrm{m}$ during the melting and drawing process for large lens radii. This results in normal hemispherical lens behaviour.

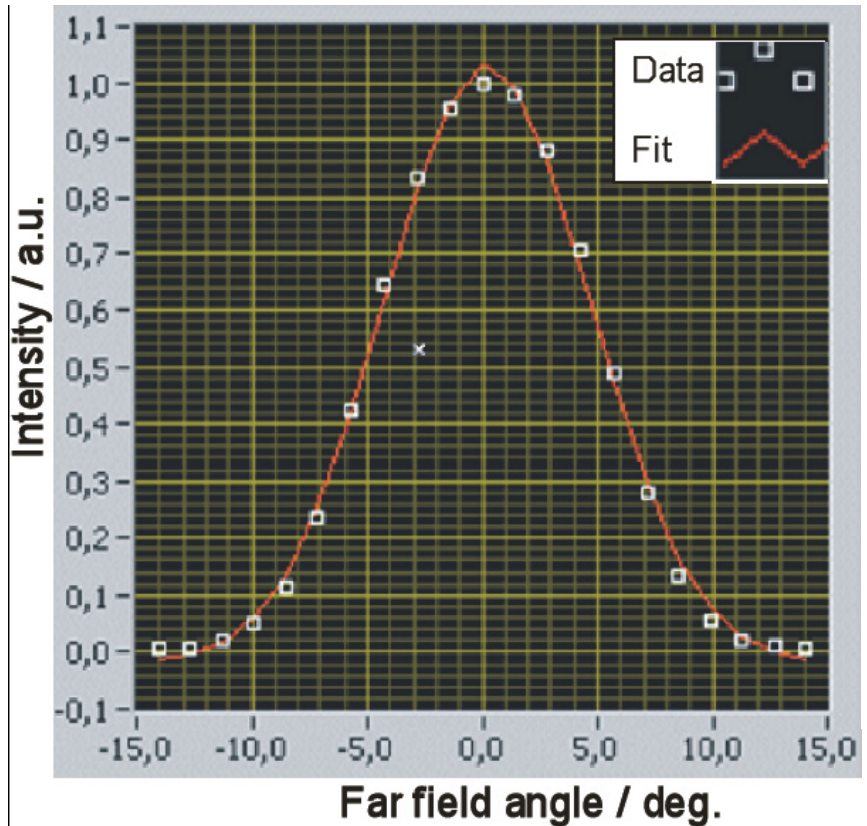

Fig. (8). Measured mode field width of NIST reference fiber [17] : $2 w_{0}=10.34 \mu \mathrm{m} \pm 3 \%$ @ $1550 \mathrm{~nm}$.

Below taper radii of $15 \mu \mathrm{m}$ the fiber core at the end of the fiber tip is highly downscaled by the drawing process, which will cause a smaller waveguide cross-section. Hereby the guiding of Gaussian lightwave in the fiber core will broaden up at its last $100 \mu \mathrm{m}$ to the fiber end. Here, the broadened optical wave will be refracted by the fiber end lens.

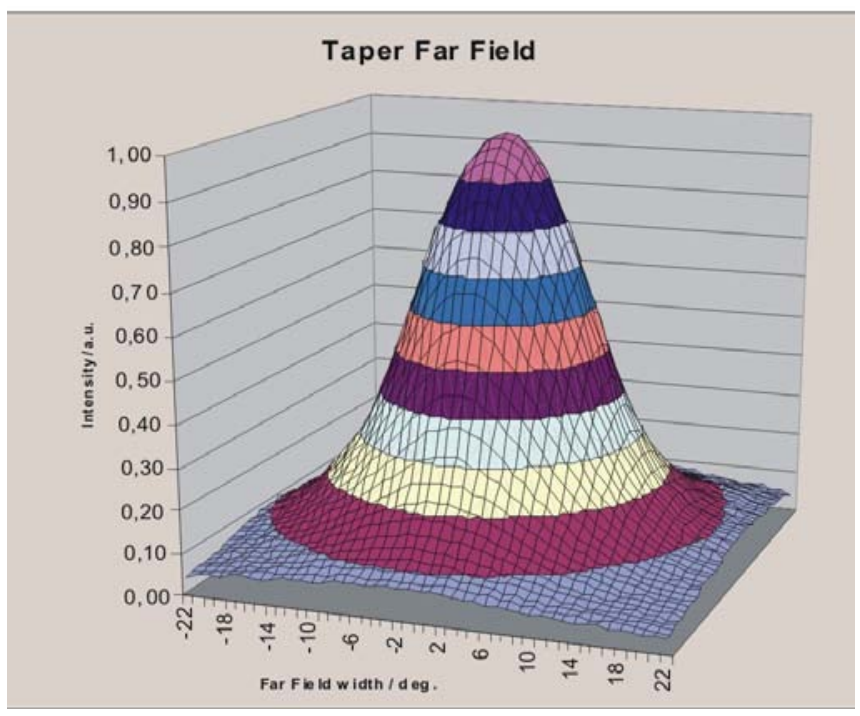

Fig. (9). Measured far field width in deg. of SMF-fiber taper with $12 \mu \mathrm{m}$ fiber end radius: $w_{0}=2.2 \mu \mathrm{m}$ (corresponding far field angle $\Theta$ $=12.64^{\circ}$ ).

The correlation between mode field radius and far field angle can be used to calculate the mode field diameter from the measured far field distribution. It can be easily calculated from Eq. (6):

$$
w_{o}=\frac{\lambda}{\pi \cdot n \cdot \tan \theta}
$$

$\lambda=$ wavelength (here $1.55 \mu \mathrm{m}$ ) and

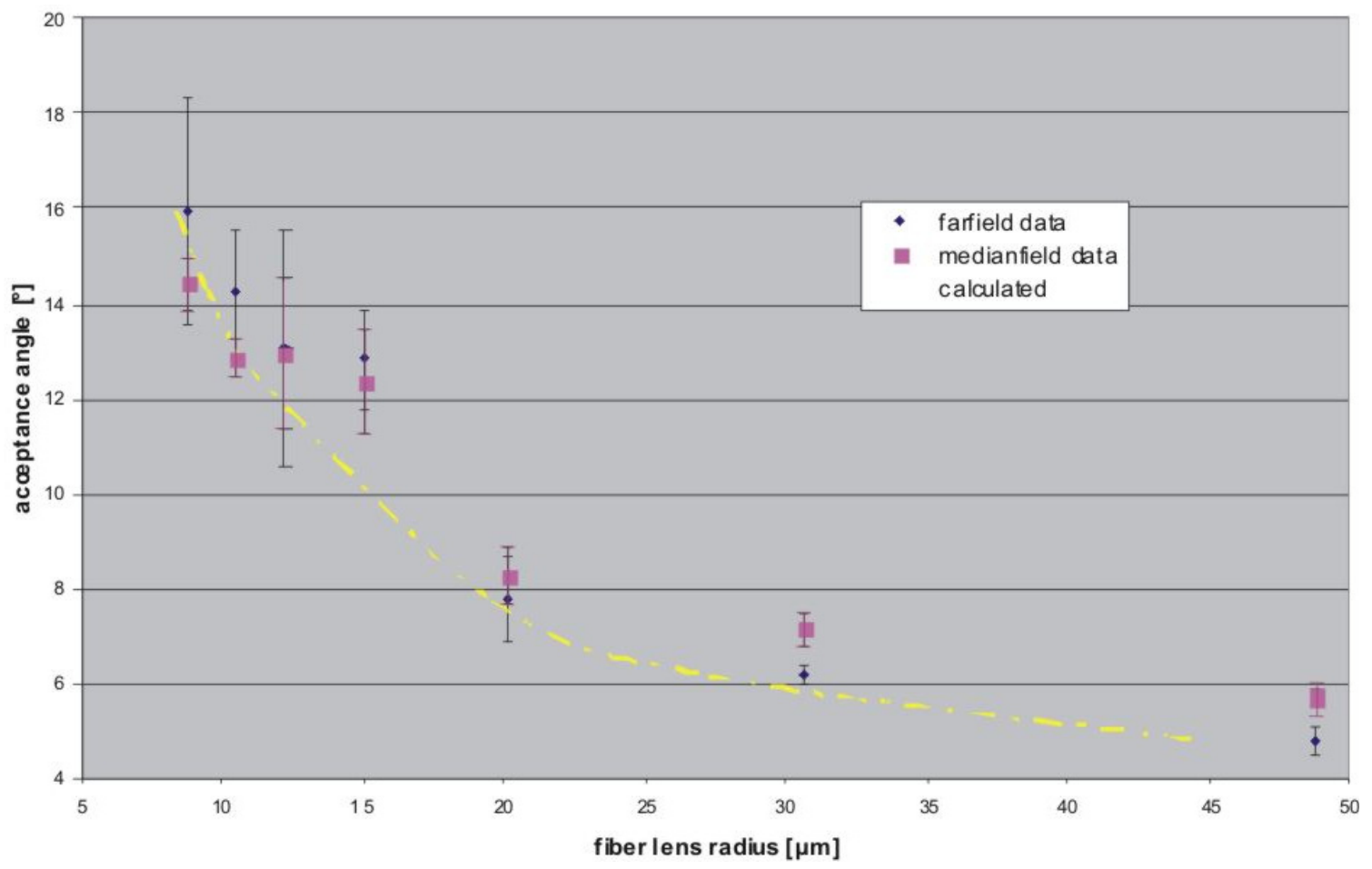

Fig. (10). Measured acceptance angle as a function of the fiber lens radius; the error bars indicate the measurement error. 
$\mathrm{n}=$ refractive index of the medium between fiber and photo receiver (here air with $\mathrm{n} \approx 1$ ).

Table 1. Fabricated SMF Lenses with Different Radii

\begin{tabular}{|c|c|c|c|c|c|c|c|}
\hline $\begin{array}{c}\text { Device/ } \\
\text { Radius } \\
(\boldsymbol{\mu m})\end{array}$ & $\mathbf{8}$ & $\mathbf{1 0}$ & $\mathbf{1 2}$ & $\mathbf{1 5}$ & $\mathbf{2 0}$ & $\mathbf{3 0}$ & $\mathbf{5 0}$ \\
\hline \hline taper 1 & 8.81 & 10.33 & 12.09 & 15.66 & 19.73 & 29.73 & 49.13 \\
\hline taper 2 & 8.53 & 10.43 & 12.35 & 15.3 & 19.99 & 29.75 & 48.76 \\
\hline taper 3 & 9.1 & 10.76 & 11.74 & 14.61 & 19.94 & 30.57 & 48.17 \\
\hline taper 4 & 8.6 & 10.38 & 12.4 & 14.31 & 20.27 & 31.35 & 49.08 \\
\hline taper 5 & 8.66 & 10.2 & 12.58 & 15.47 & 20.42 & 31.93 & 48.75 \\
\hline Mean & 8.74 & 10.42 & 12.23 & 15.07 & 20.07 & 30.07 & 48.78 \\
\hline$\sigma$ & 0.22 & 0.2 & 0.32 & 0.58 & 0.27 & 0.97 & 0.38 \\
\hline
\end{tabular}

To complete the characterization of the fiber tapers, we measured also the coupling efficiency between a typical DFB Laser diode and several self produced fiber tapers with $10 \mu \mathrm{m}$ radius. Coupling losses from $7.5 \mathrm{~dB}$ (efficiency $17.7 \%$ ) to $5.6 \mathrm{~dB}$ (efficiency $27.5 \%$ ) in combination with a DFB-laser with a strong asymmetric field distribution $\left(1550 \mathrm{~nm}\right.$, field width $w_{0}=2,2 \mu \mathrm{m}$ perpendicular, $3,5 \mu \mathrm{m}$ vertical) could be obtained with these fiber tapers which is depicted for a sample of 29 devices in Fig. (11).

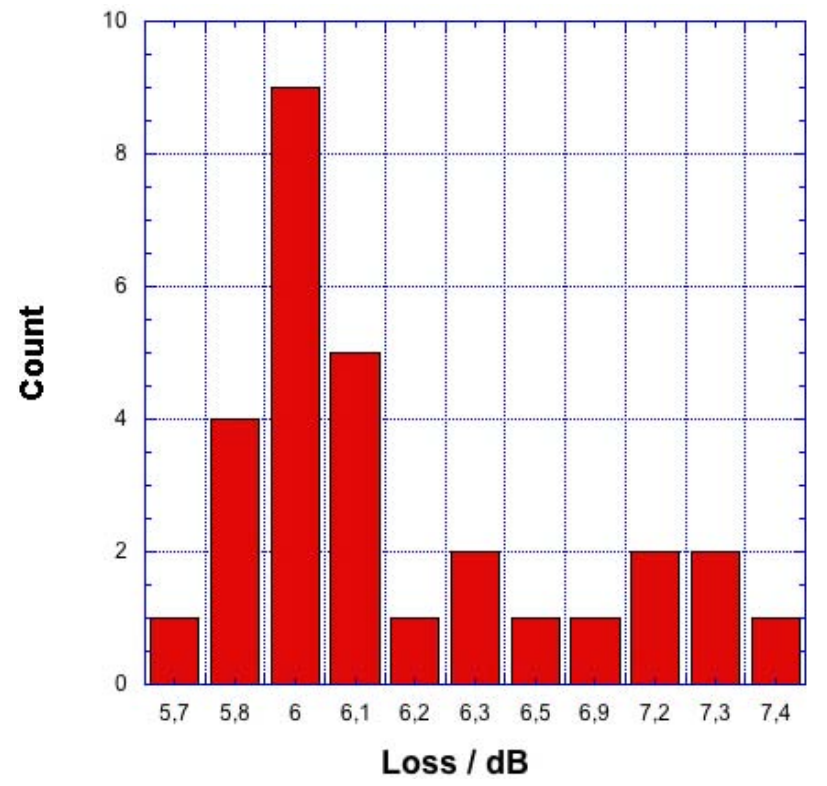

Fig. (11). Coupling losses of tapered fibers to DFB-laser.

The theoretical maximum coupling efficiency of $36.3 \%$ or $4.4 \mathrm{~dB}$ loss can be achieved between this Laser diode and an ideal adapted lensed fiber. The difference of $-1.2 \mathrm{~dB}$ between theoretical and measured data can be caused by additional coupling losses due to misalignment of the fiber to the chip waveguide.

\section{SUMMARY}

We presented a new method for the fabrication of optical mode field transformers for efficient packaging of lightwave modules. We have shown that the developed fiber lenses for SMF and SI or GI-MMF have an excellent reproducibility in mechanical and optical sizes within a very small deviation of the drawn lens radii of $0.2-1 \mu \mathrm{m}$. The optical mode fields of these lenses can be concentrated into a spot size down to $3.4 \mu \mathrm{m}$ that realizes a good field adaptation to Laser diodes. The lenses have been deeply characterized by optical and mechanical parameters.

\section{ACKNOWLEDGEMENT}

The Department of Research and Development of the Federal Republic of Germany and the Ministry of Culture of Saxony-Anhalt supported this work.

\section{REFERENCES}

[1] Naito T. One Terabit/s Transmission over $10.000 \mathrm{~km}$ using C-Band and L-Band: Networks and Optical Communications; June 2000; IOS Press, Amsterdam, The Netherlands; 2000.

[2] Fischer UHP, Zech S, Peters K. Transmitter modules with reusable fiber-chip coupling method for optical communications systems. TechOnline 2002. 21 October 2008: Available from: http://www. techonline.com/community/ed_resource/tech_paper/14811

[3] Saruwatari M, Nawata K. Semiconductor laser to single-mode coupler. Appl Opt 1979; 18: 324-31.

[4] Saruwatari M, Sugie T. Efficient laser diode to to single-mode fiber coupling using a combination of two lenses in confocal condition. IEEE J Quant Opt 1981; 18: 1347-56.

[5] Fischer UHP. Optoelectronic Packaging. VDE-Verlag: Berlin, 2002.

[6] Fischer UHP, Krips O, Müller E, Jacob A. Laser micro welding for fiber-chip coupling modules with tapered SMF-fiber ends for optical communication systems. Opt Eng 2002; 41: 3221-9.

[7] Presby M, Edwards C. Efficient Coupling of PolarisationMaintaining Fiber to Laser Diodes. IEEE Phot Tech Lett 1992; 4: 897-9.

[8] Mathyssek K, Wittmann J, Keil R. Fabrication and Investigation of Drawn Fiber Tapers with Spherical Microlenses. J Opt Commun 1985; 6: 142-6.

[9] Fischer UHP, Peters K, Ziegler R, et al. Packaging of OEIC's with Tapered Fibers for Optical Communications Systems with up to 45GHz Modulation Bandwidth: Broadband Access and Technology; June 1999; IOS Press, Berlin, Germany, 1999.

[10] Output Far-Field Radiation Pattern Measurement. Electronic Industries Association Standard RS-455-47; 1983.

[11] Firester AH, Heller ME, Sheng P. Knife-edge scanning measurements of subwavelength focused light beams. Appl Opt 1977; 16: 5605-19.

[12] Anderson W, Philen D. Spot Size Measurements for Single-Mode Fibers - A Comparison of Four Techniques. J Lightw Tech 1983; LT-1: 20-6.

[13] TIA Fiberoptic Test Procedures FOTP-191; 1998.

[14] Young M. Mode-field diameter of single-mode optical fiber by farfield scanning. Appl Opt 1998; 37(24): 5605-9.

[15] DIN32562. 21 October 2008: Available from: http://www.vde.de

[16] Windel T, Fischer UHP. Medianfield-methode - a planar method to obtain the spot-size of single mode optical components: Proceedings of LANE, September 2004; Erlangen, Germany, 2004.

[17] NIST Standard SMF fiber 2002. 21 October 2008: Available from: https://srmors.nist.gov/view_detail.cfm?srm=2513

[18] Ladany I. Laser to single-mode fiber coupling in the laboratory. Appl Opt 1993; 32: 3323-6. 